\title{
A Novel Chemical Solution to Demineralize Valvular and Coronary Calcification: Insights from Yogurt and Honey
}

Mohamad H Yamani ${ }^{1 *}$ and Raouf E Nakhleh ${ }^{2}$

${ }^{1}$ Department of Cardiovascular Medicine, Mayo Clinic Florida, USA

${ }^{2}$ Department of Anatomic pathology, Mayo Clinic Florida, USA

\begin{abstract}
Objectives: The purpose of the study is to evaluate the demineralizing efficacy of combined $L(+)$ Lactic Acid And D-Gluconic Acid (LAGA) solution and combined yogurt and honey on human specimens of calcific valves and coronaries retrieved from patients who underwent aortic valve replacement, mitral valve replacement and heart transplantation.

Background: Cardiovascular calcification is a major health problem in the aging population and hence, the need for therapeutic strategies to attenuate the disease process.

Methods: Serial sections of 4 Formalin Fixed Paraffin Embedded (FFPE) calcific aortic valves, 4 calcific mitral valves and 12 coronary arteries were treated with LAGA solution. Von Kossa silver staining was used for histological semiquantitative evaluation of extent of calcification. A direct treatment of the tissue specimens with combined yogurt and honey was also performed

Results: A complete dissolution of the calcification was noted in all the aortic, mitral and coronary specimens after treatment with LAGA solution $(\mathrm{P}<0.0001)$. A direct treatment of the aortic, mitral, and coronary specimens with combined yogurt and honey for 24 hours showed mild reduction in the degree of calcification by at least one grade that was statistically significant $(P<0.01)$.

Conclusions: LAGA solution is efficacious in demineralizing calcific aortic and mitral valves and calcific coronary arteries. Combined yogurt and honey result in mild reduction in calcification. Our study may pave the way for epidemiological studies and potential therapeutic strategies to treat patients with vascular calcification.
\end{abstract}

Keywords: Calcification; Coronary artery disease; Valvular heart disease; Yogurt; Honey; L+ lactic acid; D-gluconic acid

Abbreviations: LAGA: Combined L (+) Lactic Acid and D-Gluconic Acid; FFPE: Formalin Fixed Paraffin Embedded; HCL: Hydrochloric Acid; EDTA: Ethylenediamine Tetra Acetic Acid

\section{Introduction}

Calcific degenerative valvular heart disease is a major health problem in industrialized nations, affecting older populations. The prevalence of degenerative valvular heart disease increases with age, reported in $13 \%$ of patients older than 75 years of age and is associated with a worse survival [1]. The Cardiovascular Health Study was the first to describe the risk factors associated with calcific aortic valve disease including age, smoking, male gender, hypertension, and an elevated low-density lipoprotein, similar to those of atherosclerosis [2]. Evidence from studies on the pathogenesis of calcific aortic valve disease supports an active disease process with lipoprotein deposition, chronic inflammation, and leaflet calcification [3]. The recent emerging evidence for active regulation of proosteogenic signaling in advanced aortic valve has led to a major paradigm shift in the understanding of pathogenesis of calcification from a passive degenerative process to an actively regulated one [4]. Similarly, coronary calcification has also been recognized recently to be an active disease process where inflammatory factors synthesized during the early stages of atherosclerosis induce the expression and activation of osteoblast-like cells localized in the arterial wall that produce calcium [5]. The incidence of coronary calcification increases with advancing age reaching more than $12 \%$ in patients older than 80 years of age and portends poor survival $[6,7]$. The link between vascular calcification and advancing age has been well illustrated in the recent Horus mummies study of ancient populations [8].
The economic and social burden of both, calcific coronary and valvular heart disease calls for the need of earlier therapeutic interventions to alter the natural course of the disease process. Hence, the search for alternative strategies that may be safe, natural and efficacious is vital to attenuate the calcific disease process. Potential chemical products that may meet such criteria are lactic and gluconic acids which have demineralization properties and are naturally present in yogurt and honey, respectively.

Lactic acid (2-Hydroxypropionic Acid) is a carboxylic acid with the chemical formula $\mathrm{C}_{3} \mathrm{H}_{6} \mathrm{O}_{3}$. It is used in a wide variety of pharmaceutical and industrial applications such as cosmetics, plastics, biomaterials, medical devices, detergents, disinfectants, household cleaning products, and food industry [9-15]. Lactic acid is found primarily in sour milk products, such as yogurt $(0.9 \%-1.1 \%)$, kefir, cottage cheeses and to a lower extent in wine $(0.01 \%-0.54 \%)$ [16-18]. Many bacterial species produce lactic acid such as Lactobacillus, Bifidobacterium, Streptococcus, Pediococcus and Leuconostoc $[19,20]$.

Gluconic acid (2,3,4,5,6-Pentahydroxycaproic Acid) is an organic

*Corresponding author: Mohamad $\mathrm{H}$ Yamani, Mayo Clinic Florida, Cardiovascular Medicine, Davis 7E, 4500 San Pablo Rd, Jacksonville, FL 32224, USA, Tel: (904)953-7278; Fax: (904)953-2911; E-mail: yamani.mohamad@mayo.edu

Received November 01, 2013; Accepted November 09, 2013; Published November 14, 2013

Citation: Yamani MH, Nakhleh RE (2013) A Novel Chemical Solution to Demineralize Valvular and Coronary Calcification: Insights from Yogurt and Honey. J Cardiovasc Dis Diagn 1: 130. doi:10.4172/2329-9517.1000130

Copyright: @ 2013 Yamani MH, et al. This is an open-access article distributed under the terms of the Creative Commons Attribution License, which permits unrestricted use, distribution, and reproduction in any medium, provided the original author and source are credited. 
compound with molecular formula $\mathrm{C}_{6} \mathrm{H}_{12} \mathrm{O}_{7}$. It has a variety of uses in the chemical, pharmaceutical, food, construction and other industries $[21,22]$. It is also used in cleaning products where it dissolves mineral deposits. Gluconic acid occurs naturally in honey $(0.17 \%-1.15 \%)$, fruit, and to a much lesser extent in wine $(0.005 \%-0.1 \%)[23,24]$. Our study is designed to test the hypothesis that the combination of $\mathrm{L}(+)$ Lactic Acid and D-gluconic Acid (LAGA) may be effective in demineralization of calcific aortic and mitral valves and calcific coronary arteries with the hope that it may serve as an impetus to stimulate further clinical research to evaluate the impact of combined yogurt and honey intake on cardiovascular outcome and/or may pave the way for the use of LAGA as a therapeutic strategy in the treatment of cardiovascular calcification.

\section{Methods}

\section{Patient population}

The study included 4 patients with severely calcific aortic valve stenosis (mean age $73 \pm 8$ years, mean aortic valve area $0.82 \pm 0.08 \mathrm{~cm}^{2}$, mean aortic mean pressure gradient $43 \pm 7 \mathrm{mmHg}$ ) who underwent aortic valve replacement; 4 patients with severely calcific degenerative mitral valve disease (mean age $74 \pm 5$ years) who underwent mitral valve replacement and 4 patients with severe coronary atherosclerotic heart disease and end-stage ischemic cardiomyopathy who underwent heart transplantation (mean age $65 \pm 5$ years). The study was approved by the institutional review board of Mayo Clinic.

\section{Immunohistochemistry staining}

Formalin Fixed Paraffin Embedded (FFPE) native valves and coronary specimens were retrieved and serial sections of each specimen were obtained and evaluated semi-quantitatively for deposits of calcium using von Kossa staining protocol where tissue sections were treated with a silver nitrate solution and the silver was deposited by replacing the calcium reduced by the strong light [25]. Bone tissue was used as positive control, at each testing, where the von Kossa stain was visualized as dark brown indicating the presence of calcification (Figure 1). The valve tissues specimens and coronary specimens were cut at $5 \mu \mathrm{m}$ sections and baseline von Kossa staining was obtained prior to any chemical treatment. Leica, a commercially available decalcifying chemical solution (a combination of 15\% Hydrochloric acid (HCL) and 5\% Ethylenediamine Tetra Acetic Acid (EDTA) by weight, Fisher scientific, Pitssburgh, PA) was used as a reference to compare the demineralizing efficacy of LAGA solution. The initial highest concentration of LAGA tested was $15 \% \mathrm{~L}(+)$ lactic acid $+5 \%$ $\mathrm{D}$-gluconic acid by weight. This concentration was comparable to the leica solution. The pathologist was blinded to the type of chemical solution used.

\section{Serial dilutions}

We started the evaluation with the calcific aortic valve specimens. Since the concentration and the effect of LAGA on calcific valves have never been explored, we tested the LAGA solution $(10 \mathrm{ml})$ at 4 different concentrations and compared the efficacy to similar dilutions of leica $(10 \mathrm{ml})$. The tissue sections were mounted on glass slides that were incubated with leica and LAGA for one hour. Two aortic valve specimens that included a total of 24 aortic valve sections (12 sections per specimen), were tested at 4 different concentrations (6 sections at each level of concentration) with von Kossa staining performed as a baseline (prior to treatment, $\mathrm{n}=8$ sections), after treatment with leica ( $\mathrm{n}=8$ sections) and after treatment with LAGA ( $\mathrm{n}=8$ sections) as follows:
1) Full concentration of the original solution (100\%): leica $(15 \%$ HCL $+5 \%$ EDTA $)$ and LAGA $(15 \% \mathrm{~L}(+)$ lactic acid + 5\% D-gluconic acid).

2) $50 \%$ concentration of the original solution: leica $(7.5 \% \mathrm{HCL}$ $+2.5 \%$ EDTA $)$ and LAGA $(7.5 \% \mathrm{~L}(+)$ lactic acid + $2.5 \%$ D-gluconic acid).

3) $10 \%$ concentration of the original solution: leica (1.5\% HCL $+0.5 \%$ EDTA $)$ and LAGA $(1.5 \% \mathrm{~L}(+)$ lactic acid + $0.5 \%$ D-gluconic acid).

4) $1 \%$ concentration of the original solution: leica $(0.15 \% \mathrm{HCL}$ $+0.05 \%$ EDTA $)$ and LAGA $(0.15 \% \mathrm{~L}(+)$ lactic acid + 0.05\% D-gluconic acid).

After determining the fact that the lowest concentration of LAGA $(0.15 \% \mathrm{~L}(+)$ lactic acid $+0.05 \% \mathrm{D}$-gluconic acid) was effective in the decalcification process, we tested 2 additional aortic valve specimens at the lowest concentration to add up the total analysis to 4 specimens. Subsequently, 4 mitral valve specimens (total of 12 sections) were tested at this lowest concentration and were evaluated with von Kossa staining as a baseline (prior to treatment, $n=4$ sections), after treatment with leica ( $\mathrm{n}=4$ sections) and after treatment with LAGA ( $\mathrm{n}=4$ sections). Finally, we evaluated 4 coronary arteries ( 2 circumflex and 2 left anterior descending arteries); 3 sections per coronary artery block was obtained and a total of serial 36 sections were tested at the same lowest concentration and were evaluated with von Kossa staining as a baseline (prior to treatment, $\mathrm{n}=12$ sections), after treatment with leica ( $\mathrm{n}=12$ sections) and after treatment with LAGA ( $\mathrm{n}=12$ sections).

\section{Combined yogurt and honey}

The encouraging decalcification results achieved with the lowest concentration of LAGA prompted us to determine the direct effect of combined plain yogurt ( 3 ounces) which has high concentrations of lactic acid, and pure raw honey (one ounce), which has high concentrations of gluconic acid) on the demineralization process. The ratio of yogurt to honey ( $3: 1$ by weight) was chosen similar to the ratio of lactic acid to gluconic acid (by weight) of the LAGA solution. Since the ideal incubation period was unknown, we did a preliminary evaluation, incubating the tissue slides with combined yogurt and

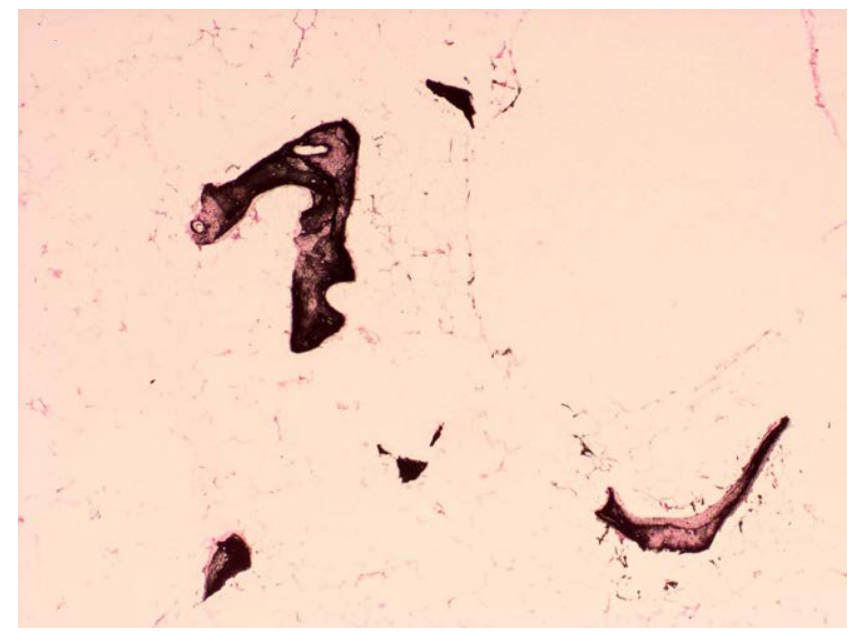

Figure 1: von Kossa staining of bone tissue. Caption: von Kossa staining of bone tissue showing dark brown discoloration of the calcium deposits. 
Citation: Yamani MH, Nakhleh RE (2013) A Novel Chemical Solution to Demineralize Valvular and Coronary Calcification: Insights from Yogurt and Honey. J Cardiovasc Dis Diagn 1: 130. doi:10.4172/2329-9517.1000130

honey for a period of one hour but no effect was demonstrated (Results are not included because of lack of efficacy). Hence, we repeated the evaluation with a 24 hour incubation period to allow adequate tissue exposure to the combined yogurt and honey (Results). The von Kossa staining was performed on serial sections of each of the aortic valves $(n=4)$, mitral valves $(n=4)$, and coronary arteries $(n=12)$ specimens as baseline (before treatment) and similar number of sections were analyzed after a 24 hour incubation with combined yogurt and honey.

\section{Statistical analysis}

The severity of calcification was graded (30) as follows:

Score $1=$ absent calcification.

Score $2=$ mild calcification .

Score $3=$ moderate calcification .

Score $4=$ moderate-severe calcification

Score $5=$ severe calcification .

Data are presented as mean \pm standard deviation. Statistical analyses were performed using the general linear mixed-model Analysis of Variance (ANOVA). The results of the aortic and mitral valve specimens tested at the lowest concentration of LAGA were combined for statistical purposes. Similarly, the results of the aortic and mitral valve specimens tested with combined yogurt and honey were also combined for statistical purposes. Differences were considered significant at $P<0.05$.

\section{Results}

\section{Impact of LAGA solution}

The demineralizing effect of LAGA on the aortic valve specimens at 4 different concentrations is illustrated in Figure 1. The baseline calcification deposits prior to treatment is shown (Figures 1a,1d,1g,1j). The effect of leica, which is known to have decalcification properties, is demonstrated (Figure $1 \mathrm{~b}\{100 \%\}$, Figure $1 \mathrm{e}\{50 \%\}$, Figure $1 \mathrm{~h}\{10 \%\}$, and Figure $1 \mathrm{k}\{1 \%\})$, showing complete dissolution of calcification. LAGA was equally efficacious in demineralizing the calcific deposits (Figure 1c $\{100 \%\}$, Figure 1f $\{50 \%\}$, Figure 1i $\{10 \%\}$, and Figure $2\{1 \%\})$ of the aortic valve specimens even at the lowest concentration, $\mathrm{L}(+)$ lactic acid $(0.15 \%)+\mathrm{D}$-gluconic acid $(0.05 \%)$ which represents $1 \%$ of the original solution concentration. The mitral valve specimens were tested at this lowest concentration and again the demineralizing efficacy of LAGA is clearly demonstrated (Figure 3 and Table 1). This lowest concentration of LAGA was also efficacious in decalcifying the coronary specimens (Figure 4 and Table 1).

\section{Impact of yogurt and honey}

The effect of combined yogurt and honey is shown in Figure 5. There was mild reduction in the amount of calcification at 24 hours in each of the calcific aortic/mitral valve and coronary specimens that were statistically significant (Table 2).
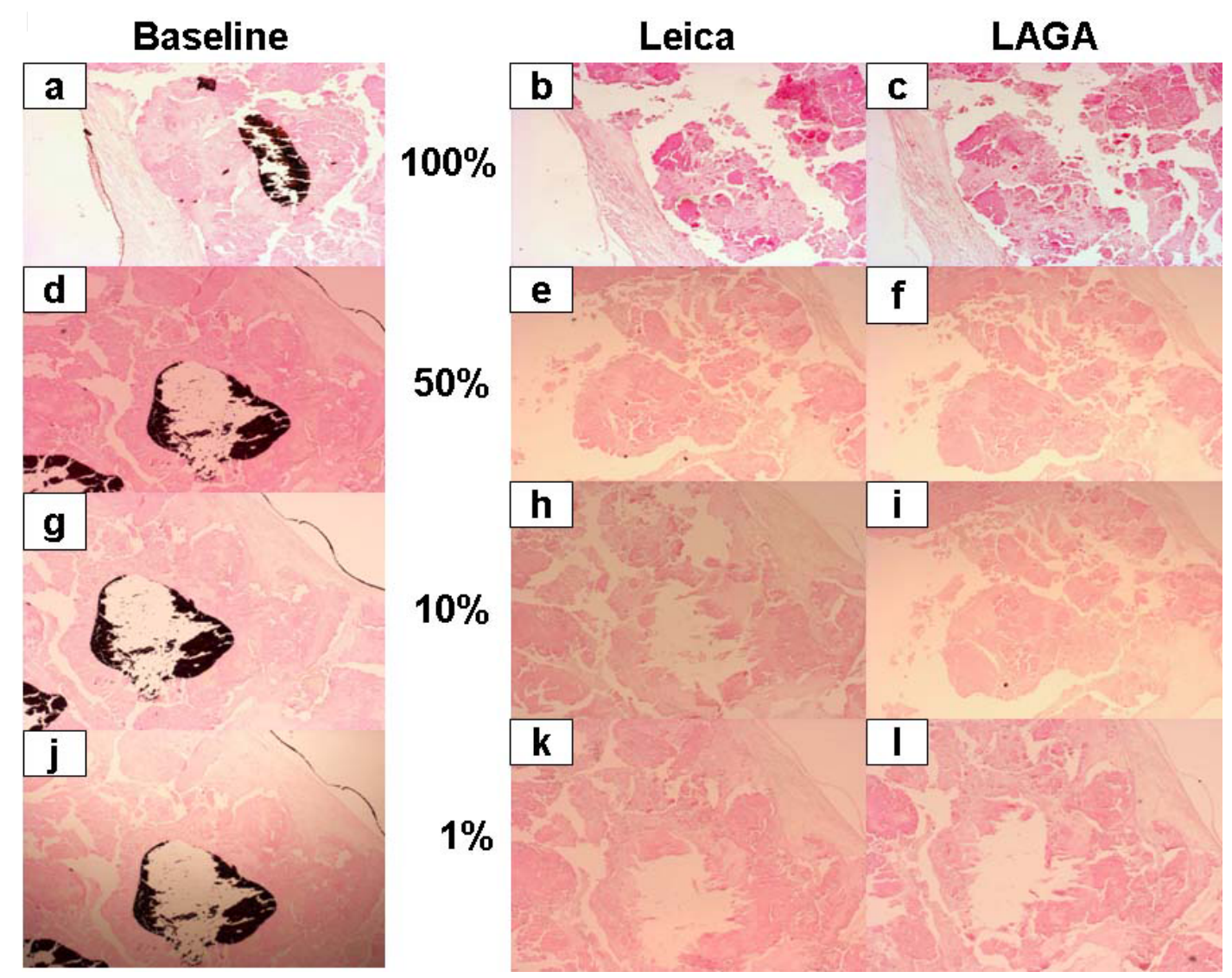

Figure 2: von Kossa staining of calcific aortic valve specimens. Caption: von Kossa staining of calcific aortic valve specimens showing baseline calcification deposits (a, d, g, and j), after treatment with lecia (b $\{100 \%\}$, e $\{50 \%\}, h\{10 \%\}$, and $\mathrm{k}\{1 \%\}$ ) and after treatment with LAGA solution (c $\{100 \%\}$, $f\{50 \%\}$, $\{10 \%\}$, and I $\{1 \%\}$ ) at 4 different concentrations. 
Baseline

a

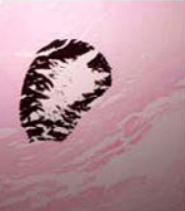

Leica

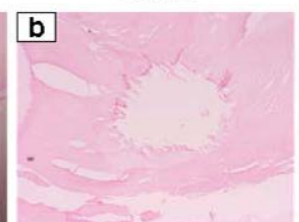

LAGA

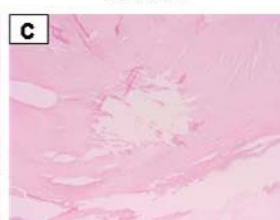

Figure 3: von Kossa staining of calcific mitral valve specimens. Caption: von Kossa staining of calcific mitral valve specimens showing baseline calcification deposits (a), after treatment with leica (b) and after treatment with LAGA (c) solution.

\begin{tabular}{|c|c|c|c|c|}
\hline Specimens & $\begin{array}{c}\text { Baseline } \\
\text { Score 1 }\end{array}$ & $\begin{array}{c}\text { Leica } \\
\text { Score 2 }\end{array}$ & $\begin{array}{c}\text { LAGA } \\
\text { Score 3 }\end{array}$ & P-value \\
\hline Mitral/aortic & $4.75 \pm 0.46(n=8)$ & $1 \pm 0(n=8)$ & $1 \pm 0(n=8)$ & $<0.00001$ \\
\hline Coronaries & $4.66 \pm 0.49(n=12)$ & $1 \pm 0(n=12)$ & $1 \pm 0(n=12)$ & $<0.00001$ \\
\hline
\end{tabular}

Score $1=$ absent calcification. Score $2=$ mild calcification. Score $3=$ moderate calcification. Score $4=$ moderate-severe calcification. Score $5=$ severe calcification

Table 1: Impact of LAGA on valvular and coronary calcification score.

\section{Discussion}

Calcification is an age-linked active complex process where oxidized low-density lipoproteins, T-lymphocyte accumulation, autophagic cell death, osteoblast-like differentiation, and osteopontin producing macrophages have been shown to play major roles in the pathogenesis of severe calcific aortic valve stenosis [4,26-30]. The initial triggering factor involved in the pathogenesis of degenerative mitral valve disease may be different, involving changes in the extracellular matrix, mediated by catabolic enzymes [31] but ultimately, calcification develops with adverse sequalea. There has also been a paradigm shift in the understanding of pathogenesis of coronary calcification from a passive atherosclerotic process to an actively regulated one involving activation of osteoblast cells and utilizing similar biochemical processes as in bone formation $[5,32,33]$. Vascular calcification has been emerged as a strong and independent risk factor for cardiovascular morbidity and mortality [34]. Surgery has been the main treatment modality in patients with severe symptomatic calcific valvular heart disease and recently, transcatheter aortic valve replacement has gained an increasing popularity as an alternative approach in patients with severe aortic stenosis who are not suitable candidates for surgery [35]. Revascularization has been the main strategy in symptomatic patients with severe calcific coronary atherosclerotic heart disease in addition to medical therapy. Since calcification is linked to aging and the latter is inevitable and irreversible, then a key question arises as to whether calcification should follow the same destiny as aging or could we alter the natural course of the disease by instituting early innovative therapeutic strategies.

Our study introduces a novel concept based on 2 chemicals, lactic acid and gluconic acid, which are naturally present in high concentration in yogurt and honey, respectively. We have shown that the chemical solution, LAGA, was as effective as the commercially available solution, leica, in decalcifying the valves and coronaries even at its lowest concentration. There has been a lot of enthusiasm in designing new therapeutic strategies to alleviate the cardiovascular aging-degenerative process, but unfortunately, it was met with disappointing results. A meta-analysis of several studies that involved the use of statins in patients with aortic stenosis failed to show any improvement in outcome or delay in progression of the severity of aortic stenosis [36]. Recently, the use of disodium-EDTA chelation

therapy in patients with coronary artery disease and prior myocardial infarction showed less coronary revascularization in the chelation arm (15\%) compared to placebo (18\%); HR, 0.81 (95\% CI, 0.64-1.02); however, the study received major critique and the findings were not sufficient to support the routine use of chelation therapy as secondary prevention for such patients [37]. Our findings may pave the way for further clinical research to determine if the LAGA solution is effective in

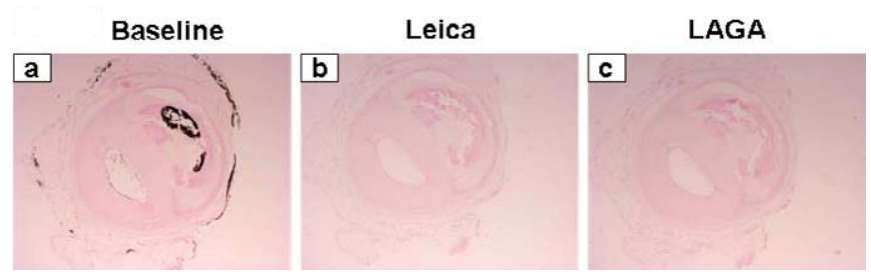

Figure 4: von Kossa staining of calcific coronary specimens. Caption: von Kossa staining of calcific coronary specimens showing baseline calcification deposits (a), after treatment with leica (b) and after treatment with LAGA (c) solution.

Baseline

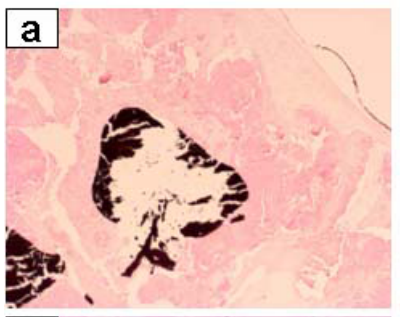

C
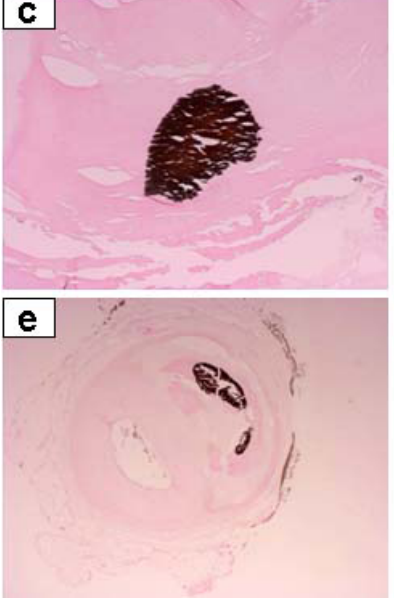

Figure 5: Impact of combined yogurt and honey on calcification. Caption von Kossa staining of calcifc aortic valve (a) mitral valve (c) and coronary specimens (e) at baseline and after 24 hour treatment with combined yogurt and honey $(b, d, f)$ showing mild reduction in the calcification process.

\begin{tabular}{|c|c|c|c|}
\hline Specimens & $\begin{array}{c}\text { Baseline } \\
\text { Score 4 }\end{array}$ & $\begin{array}{c}\text { Yogurt + Honey } \\
\text { Score 5 }\end{array}$ & P-value \\
\hline Mitral/aortic & $4.87 \pm 0.36(n=8)$ & $4.12 \pm 0.35(n=8)$ & 0.001 \\
\hline Coronaries & $4.58 \pm 0.51(n=12)$ & $4.08 \pm 0.29(n=12)$ & 0.007 \\
\hline
\end{tabular}

Score $1=$ absent calcification. Score $2=$ mild calcification. Score $3=$ moderate calcification. Score 4= moderate-severe calcification. Score $5=$ severe calcification

Table 2: Impact of combined yogurt and honey on valvular and coronary calcification score. 
delaying the progression of degenerative valvular disease and vascular calcification. Naturally, this will require initially a pharmacodynamic/ safety study followed by a large multicenter clinical trial with a careful design, selecting the appropriate timing of initiation of treatment, to determine if LAGA would alter the natural course of the disease and result in any favorable impact on cardiovascular outcome.

Another novel major finding of the study was that direct use of combined yogurt and honey showed some efficacy in decalcifying the valves and coronaries. Turkey has the largest yogurt consumption per capita ( $45 \mathrm{~kg}$ per year) and Australia has the largest honey consumption per capita (1.6 kg per year) in the world. Unfortunately, there is a paucity of epidemiological data in the literature about the impact of consumption of yogurt and honey on cardiovascular outcome. A non-randomized Australian study in elderly women, has shown that consumption of yogurt (but not cheese or milk) more than 100 $\mathrm{gm} / \mathrm{d}$ was associated with a significantly lower common carotid artery intima-media thickness than did participants with lower consumption (unadjusted $=-0.024 \mathrm{~mm}, \mathrm{P}=0.002$ ) [38]. This relation remained significant after adjustment for baseline, dietary, and lifestyle risk factors (multivariable analysis $=-0.023 \mathrm{~mm}, \mathrm{P}=0.003$ ). A recent randomized placebo-controlled multicenter trial has shown that consumption of a two daily servings of $150 \mathrm{~g}$ of low-fat yoghurt supplemented with whey malleable protein matrix over a 3 month period resulted in a significantly larger reduction of triglycerides concentrations in comparison to placebo (relative change $-16 \%, \mathrm{P}=0.004$ ) [39]. The relative treatment difference in fasting plasma glucose was $-7.1 \mathrm{mg} /$ $\mathrm{dl}(\mathrm{P}=0.089)$ and this effect was more pronounced in patients with impaired fasting glucose at baseline $(-11 \mathrm{mg} / \mathrm{dl}, \mathrm{P}=0.03)$. Further, in patients with hypertension, the relative treatment difference in systolic $\mathrm{BP}$ reached $-5.9 \mathrm{mmHg}(\mathrm{P}=0.054)$ and the relative treatment difference in body weight was $-1.7 \mathrm{~kg}(\mathrm{P}=0.015)$. Hence, such a dietary intervention has a significant impact on the metabolic syndrome and may potentially improve cardiovascular risk factors. Honey intake has also shown some promising results as evidenced in a randomized clinical trial where daily consumption of $70 \mathrm{gm}$ of honey for one month resulted in significant reduction in total cholesterol by 3.3\%, LDL-C by $4.3 \%$, triacylglycerole by $19 \%$, and CRP by $3.3 \%(\mathrm{p}<0.05)$ in patients with cardiovascular risk factors [40]. These trials are encouraging but they are small and do not address clinical cardiovascular outcome. It is possible that yogurt and honey may impact cardiovascular outcome through an effect on metabolic syndrome and the process of atherosclerosis, but epidemiological studies are required to learn more about the association of such a dietary intervention with outcome and randomized clinical trials to further determine if yogurt/honey intake will result in a favorable outcome.

\section{Limitations}

Our study has some limitations. Our study introduces a novel concept but it does not address the mechanisms of calcification as it is beyond the scope of this study and hence, our study is mainly an observational one, though, with significant implications. We did not test the effect of lactic acid and gluconic acid separately because we were trying to find a novel solution that mimicks the chemicals found in yogurt and honey as a combined product. The tissue specimens studied were specifically selected for the purpose of calcification evaluation and hence, our results may not necessarily apply to congenital or rheumatic valvular heart disease or non-calcific coronary atherosclerotic heart disease. The evaluation was semiquantitative in nature. This may not influence the results of the impact of LAGA solution because there was complete dissolution of calcification, but a quantitative calcium determination would be helpful to better evaluate the exact degree of reduction of calcification when evaluating the impact of combined yogurt and honey. However, we did observe semiquantitative changes that were mild but statistically significant.

\section{Conclusions}

Our study shows that LAGA solution at a low concentration $(0.15 \%$ $\mathrm{L}(+)$ lactic acid $+0.05 \% \mathrm{D}$-gluconic acid) is effective in dissolving the calcification deposits in calcific aortic valves, mitral valves and coronary arteries and that combined yogurt and honey may have a direct impact on valvular and vascular calcification. Further translational and epidemiological research is required to verify if such a biochemical event would be translated into a favorable clinical cardiovascular outcome.

\section{Acknowledgments}

The authors acknowledge the technical support of Ariston Librero.

\section{References}

1. Nkomo VT, Gardin JM, Skelton TN, Gottdiener JS, Scott CG, et al. (2006) Burden of valvular heart diseases: a population-based study. Lancet 368 : 1005-1011.

2. Stewart BF, Siscovick D, Lind BK, Gardin JM, Gottdiener JS, et al. (1997) Clinical factors associated with calcific aortic valve disease. Cardiovascular Health Study. J Am Coll Cardiol 29: 630-634.

3. Freeman RV, Otto CM (2005) Spectrum of calcific aortic valve disease: pathogenesis, disease progression, and treatment strategies. Circulation 111: 3316-3326.

4. Miller JD, Weiss RM, Serrano KM, Castaneda LE, Brooks RM, et al. (2010) Evidence for active regulation of pro-osteogenic signaling in advanced aortic valve disease. Arterioscler Thromb Vasc Biol 30: 2482-2486.

5. Serrano CV Jr, Oranges M, Brunaldi V, de M Soeiro A, Torres TA, et al. (2011) Skeletonized coronary arteries: pathophysiological and clinical aspects of vascular calcification. Vasc Health Risk Manag 7: 143-151.

6. McClelland RL, Chung H, Detrano R, Post W, Kronmal RA (2006) Distribution of coronary artery calcium by race, gender, and age: results from the MultiEthnic Study of Atherosclerosis (MESA). Circulation 113: 30-37.

7. Budoff MJ, Shaw LJ, Liu ST, Weinstein SR, Mosler TP, et al. (2007) Long-term prognosis associated with coronary calcification: observations from a registry of 25,253 patients. J Am Coll Cardiol 49: 1860-1870.

8. Thompson RC, Allam AH, Lombardi GP, Wann LS, Sutherland ML, et al. (2013) Atherosclerosis across 4000 years of human history: the Horus study of four ancient populations. Lancet 381: 1211-1222.

9. Smith WP (1996) Epidermal and dermal effects of topical lactic acid. J Am Acad Dermatol 35: 388-391.

10. Venkitanarayanan KS, Lin CM, Bailey H, Doyle MP (2002) Inactivation of Escherichia coli $\mathrm{O} 157: \mathrm{H} 7$, Salmonella enteritidis, and Listeria monocytogenes on apples, oranges, and tomatoes by lactic acid with hydrogen peroxide. $J$ Food Prot 65: 100-105.

11. Shastri VP, Martin I, Langer R (2000) Macroporous polymer foams by hydrocarbon templating. Proc Natl Acad Sci U S A 97: 1970-1975.

12. Mazzocchetti L, Sandri S, Scandola M, Bergia A, Zuccheri G (2007) Radiopaque organic-inorganic hybrids based on poly(D,L-lactide). Biomacromolecules 8 : 672-678.

13. Hossain MM, Maisuria JL (2008) Effects of organic phase, fermentation media and operating conditions on lactic Acid extraction. Biotechnol Prog 24: 757-765.

14. Burger C, Chu B (2007) Functional nanofibrous scaffolds for bone reconstruction. Colloids Surf B Biointerfaces 56: 134-141.

15. Divya JB, Varsha KK, Nampoothiri KM (2012) Newly isolated lactic acid bacteria with probiotic features for potential application in food industry. Appl Biochem Biotechnol 167: 1314-1324.

16. Cruz AG, Castro WF, Faria JA, Lollo PC, Amaya-Farfán J, et al. (2012) Probiotic yogurts manufactured with increased glucose oxidase levels: postacidification 
Citation: Yamani MH, Nakhleh RE (2013) A Novel Chemical Solution to Demineralize Valvular and Coronary Calcification: Insights from Yogurt and Honey. J Cardiovasc Dis Diagn 1: 130. doi:10.4172/2329-9517.1000130

proteolytic patterns, survival of probiotic microorganisms, production of organic acid and aroma compounds. J Dairy Sci 95: 2261-2269.

17. Adolfsson O, Meydani SN, Russell RM (2004) Yogurt and gut function. Am J Clin Nutr 80: 245-256.

18. Petri A, Pfannebecker J, Fröhlich J, König H (2013) Fast identification of wine related lactic acid bacteria by multiplex PCR. Food Microbiol 33: 48-54.

19. Mancini A, Lazzi C, Bernini V, Neviani E, Gatti M (2012) Identification of dairy lactic acid bacteria by tRNAAla-23S rDNA-RFLP. J Microbiol Methods 91: 380-

20. Berlec A, Ravnikar M, Strukelj B (2012) Lactic acid bacteria as oral delivery systems for biomolecules. Pharmazie 67: 891-898.

21. Anastassiadis S, Morgunov IG (2007) Gluconic acid production. Recent Pat Biotechnol 1: 167-180.

22. Ramachandran S, Fontanille P, Pandey A, Larroche C (2006) Gluconic Acid: Properties, Applications and Microbial Production. Biotechnol 44: 185-195.

23. Daniele G, Maitre D, Casabianca H (2012) Identification, quantification and carbon stable isotopes determinations of organic acids in monofloral honeys. A powerful tool for botanical and authenticity control. Rapid Commun Mass Spectrom 26: 1993-1998.

24. Campuzano S, Gamella M, Serra B, Reviejo AJ, Pingarrón JM (2007) Integrated electrochemical gluconic acid biosensor based on self-assembled monolayermodified gold electrodes. Application to the analysis of gluconic acid in musts and wines. J Agric Food Chem 55: 2109-2114.

25. Bills CE, Eisenberg H, Pallante SL (1974) Complexes of organic acids with calcium phosphate: the Von Kossa stain as a clue to the composition of bone mineral. Johns Hopkins Med J 128: 194-207.

26. Olsson M, Thyberg J, Nilsson J (1999) Presence of oxidized low density lipoprotein in nonrheumatic stenotic aortic valves. Arterioscler Thromb Vasc Biol 19: 1218-1222.

27. O'Brien KD, Kuusisto J, Reichenbach DD, Ferguson M, Giachelli C, et al. (1995) Osteopontin is expressed in human aortic valvular lesions. Circulation 92: 2163-2168.

28. Mohler ER 3rd, Adam LP, McClelland P, Graham L, Hathaway DR (1997) Detection of osteopontin in calcified human aortic valves. Arterioscler Thromb Vasc Biol 17: 547-552.

29. Rajamannan NM, Subramaniam M, Rickard D, Stock SR, Donovan J, et al (2003) Human aortic valve calcification is associated with an osteoblast phenotype. Circulation 107: 2181-2184.
30. Somers $\mathrm{P}$, Knaapen M, Kockx M, van Cauwelaert $\mathrm{P}$, Bortier $\mathrm{H}$, et al. (2006) Histological evaluation of autophagic cell death in calcified aortic valve stenosis. J Heart Valve Dis 15: 43-47.

31. Rabkin E, Aikawa M, Stone JR, Fukumoto Y, Libby P, et al. (2001) Activated interstitial myofibroblasts express catabolic enzymes and mediate matrix remodeling in myxomatous heart valves. Circulation 104: 2525-2532.

32. Shanahan CM, Cary NR, Metcalfe JC, Weissberg PL (1994) High expression of genes for calcification-regulating proteins in human atherosclerotic plaques. J Clin Invest 93: 2393-2402.

33. Dhore CR, Cleutjens JP, Lutgens E, Cleutjens KB, Geusens PP, et al (2001) Differential expression of bone matrix regulatory proteins in human atherosclerotic plaques. Arterioscler Thromb Vasc Biol 21: 1998-2003.

34. Chatrou ML, Winckers K, Hackeng TM, Reutelingsperger CP, Schurgers LJ (2012) Vascular calcification: the price to pay for anticoagulation therapy with vitamin K-antagonists. Blood Rev 26: 155-166.

35. Makkar RR, Fontana GP, Jilaihawi H, Kapadia S, Pichard AD, et al. (2012) PARTNER Trial Investigators. Transcatheter Aortic-Valve Replacement for Inoperable Severe Aortic Stenosis. N Engl J Med 366: 1696-1704.

36. Parolari A, Tremoli E, Cavallotti L, Trezzi M, Kassem S, et al. (2011) Do statins improve outcomes and delay the progression of non-rheumatic calcific aortic stenosis? Heart 97: 523-529.

37. Lamas GA, Goertz C, Boineau R, Mark DB, Rozema T, et al. (2013) Effect of disodium EDTA chelation regimen on cardiovascular events in patients with previous myocardial infarction: the TACT randomized trial. JAMA 309: 1241 1250.

38. Ivey KL, Lewis JR, Hodgson JM, Zhu K, Dhaliwal SS, et al. (2011) Association between yogurt, milk, and cheese consumption and common carotid artery intima-media thickness and cardiovascular disease risk factors in elderly women. Am J Clin Nutr 94: 234-239.

39. Gouni-Berthold I, Schulte DM, Krone W, Lapointe JF, Lemieux P, et al. (2012) The whey fermentation product malleable protein matrix decreases TAG concentrations in patients with the metabolic syndrome: a randomised placebocontrolled trial. Br J Nut 107: 1694-1706.

40. Yaghoobi N, Al-Waili N, Ghayour-Mobarhan M, Parizadeh SM, Abasalti Z, et al. (2008) Natural honey and cardiovascular risk factors; effects on blood glucose cholesterol, triacylglycerole, CRP, and body weight compared with sucrose. ScientificWorldJournal 8: 463-469. 